\title{
An Alternate Sunscreen: Taraxacum officinale
}

\author{
Simra Mirza \\ Institute of Knowledge 1009 Vía Sorella, Diamond Bar, CA 91789, U.S.A.; smirza@iokschool.com
}

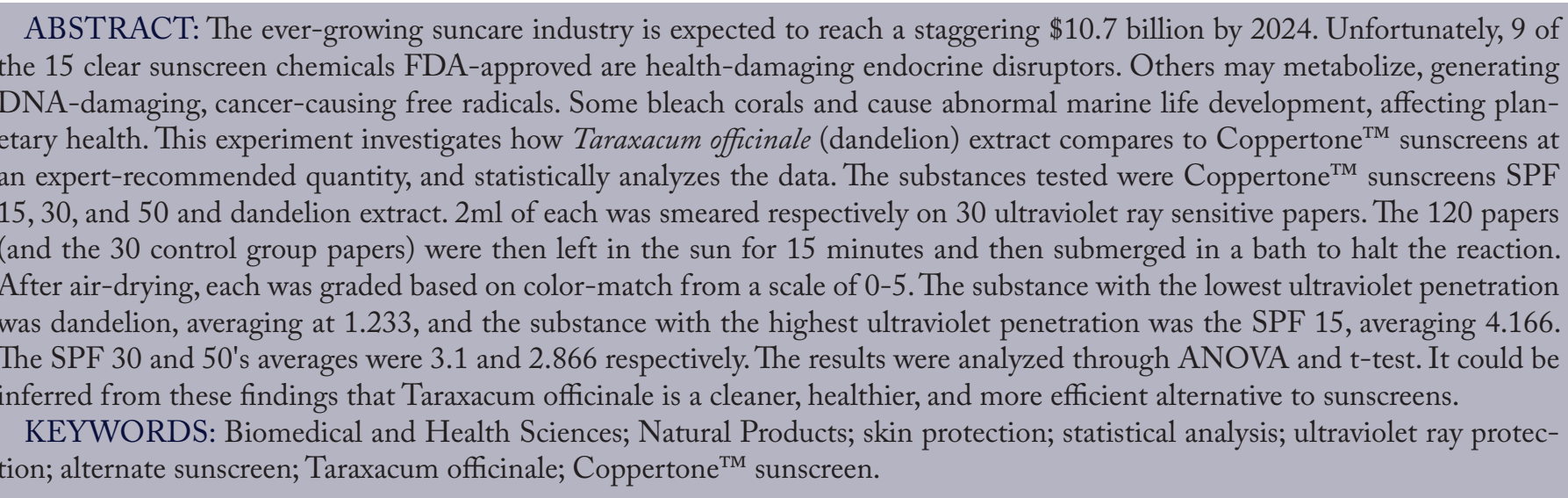

\section{Introduction}

Due to increasing global awareness regarding the havoc that unprotected sun exposure can have on our skin, sunscreen lotions have become one of the fastest-growing aspects of the skincare industry. ${ }^{1}$ As a matter of fact, in 2013, at a global scale, the sun care market generated 5.6 billion in US dollars and according to experts, is predicted to reach a staggering amount of 10.7 billion by $2024 .^{2}$ For nearly a hundred years, sunscreens have existed. They were created with the goal to block out the sun. Starting out, they were ashen substances made with zinc oxide that no one was keen on using, but scientists rose to the challenge and were able to create a sunscreen with clear chemicals. In the year 1944, Coppertone brand sunscreens became the first mass marketed sunscreen that people willingly used. ${ }^{3}$ Studies have shown that unprotected ultraviolet (UV) ray exposure causes skin cancer and ages the skin prematurely, which highlights the importance of guarding our skin against it. ${ }^{3}$ There are three components to ultraviolet irradiation: ultraviolet A (UVA), ultraviolet B (UVB), and ultraviolet C (UVC). Whereas UVC is almost entirely absorbed by the Earth's ozone layer, UVA and UVB still reach the Earth in amounts that are damaging to our skin. UVB in particular is more of a threat, as it penetrates the epidermis and the upper section of the dermis, damaging fibroblast cells, leading to photoaging, sunburn, and skin cancer.

Within the industry, there are two types of sunscreens: physical (also called mineral or inorganic) and chemical (also called organic). Physical sunscreens reflect UVA and UVB rays and the two most common ingredients for this particular branch of sunscreens are titanium dioxide and zinc oxide. As for chemical sunscreens, they absorb the UV rays and prevent them from penetrating the skin and causing damage. These sunscreens often include actives such as oxybenzone, avobenzone, and octinoxate, to name a few main ones.

There are seventeen individual ingredients approved by the Food and Drug Administration for use as actives in sunscreens, and fifteen of them are clear chemicals that absorb UV rays. However, nine of these fifteen are known endocrine disruptors that are chemicals that disrupt the function of human hormones which can be detrimental to our health and overall quality of life. Endocrine disruptors can cause the abnormal development of fetuses and growing children as well as low sperm count, infertility, and early puberty. Some can contribute to breast and ovarian cancer development and increase the chances of prostate cancer. The endocrine disruptors find their way into our bodies due to the nature of the sunscreen, being that it needs to be rubbed in. When they are rubbed in, they don't sit directly on the skin, but rather sink in and are absorbed by the skin and swiftly reach the bloodstream. They can be detected in urine, breast milk, and blood at alarming levels for up to 48 hours after one application. This is because they scatter throughout the body, evading detoxification by the liver. What's worse is that reapplication is necessary for them--some even lose $90 \%$ of their effectiveness within an hour. ${ }^{3}$

Additionally, because chemical sunscreens work by absorbing ultraviolet rays, some may mutate after being used up. Some even produce DNA-damaging chemicals referred to as free radicals which can lead to cancers. ${ }^{3}$ Moreover, according to EWG, 80 percent of sunscreens contain oxybenzone. 4 Unfortunately, sunscreen ingredients such as oxybenzone, benzophenone, and octocrylene are potentially skin carcinogenic or enter our bodies through our skin and contain other health risks. ${ }^{5}$

One may suggest using physical sunscreens and forgoing chemical sunscreens if they pose a health risk--however, these physical sunscreens have a detrimental effect on the health of 
not only humans, but the planet. As mentioned, zinc oxide is a main physical sunscreen active ingredient and humans pile on physical sunscreens containing it and go for a swim at the beach, causing it to wash off their skin and transfer into the water. Mounting research on the subject suggests that zinc oxide causes severe coral bleaching, which damages both hard corals and their symbiotic algae. ${ }^{6}$ Unfortunately, this problem regarding sunscreens and the marine environment is not limited to physical sunscreens; chemical sunscreen actives such as oxybenzone also have harmful effects. One study showed that oxybenzone began causing severe damage to corals at the low concentration of a drop of water in six-and-a-half Olympic-sized swimming pools. ${ }^{7}$ Another study from 2016 suggested that oxybenzone caused an increase in bleaching susceptibility, genotoxicity, abnormal skeleton growth due to endocrine disruption, and deformation in baby coral. ${ }^{8}$ The damage from sunscreen chemicals coupled with climate change spells disaster for corals and other marine life forms. Because 6,000 tons of sunscreen lotion washes off of tourists' skin into Hawaii's waters, the State of Hawaii passed a bill in May of 2018 that would ban sunscreens with oxybenzone and octinoxate, which took effect in January of $2021 .{ }^{9}$

In response to this bill and the overall uproar surrounding such active ingredients in sunscreens, companies began creating and marketing sunscreens as "reef-safe" by forgoing oxybenzone. However, these sunscreen companies use chemicals such as octocrylene, homosalate, and octisalate to replace it, which may be harmful to marine life. For example, one study in Science of the Total Environment found that octocrylene may adversely affect liver and brain development in zebrafish, making it notso "reef-safe" as it affects other forms of marine life instead. In fact, octocrylene has been detected--along with oxybenzone, octinoxate, etc.--in numerous fish species globally. ${ }^{10}$ Due to the effects of both physical and chemical sunscreens on the health of humans and the planet, a healthier, environmentallyfriendly, and efficient protection against UV rays is needed.

Recently, interest in herbal medicine and its research has grown at a global scale, as various herbal extracts are being shown to possess beneficial properties. Dandelion, or Taraxacum officinale, is found in almost every region on Earth. It has been shown to possess many properties including being anticarcinogenic, antioxidant, and anti-inflammatory. ${ }^{11}$ For centuries, dandelion extracts have been used in traditional Chinese and Native American medicine in treatments for cancers, hepatitis, and a variety of digestive diseases. ${ }^{5}$ Studies suggest that ingesting them may help fight inflammation, cut cancer risk, aid diabetes treatment, promote heart health, help in weight loss, promote bone health, improve liver health, boost immunity, prevent anemia, prevent water retention in kidneys, and help boost skin health. ${ }^{12,13}$ In addition, due to their high Vitamin $\mathrm{C}$ content, they are a great topical treatment in combating free radicals (that can be caused by chemical sunscreens) and treating aging skin. Due to its high alkaline levels, it can be useful in combating itching, eczema, and the bacteria that cause acne. ${ }^{14}$

Previous research suggests that dandelion extract possesses UV blocking capabilities. ${ }^{15}$ In the previous study, 0.6 ounces of all the potential sun blocking substances were used to acquire results. However, the amount of sunscreen that is recommended by experts to use for an entire face is only a nickel-sized amount. ${ }^{16}$ A nickel-sized amount is approximately $2 \mathrm{~mL}$, or 0.067628 ounces. Thus, the measurements used to obtain results in the study were not truly reflective of the amount a person would use, which is why, in this experiment, the efficacy of dandelion extracts and Coppertone ${ }^{\mathrm{TM}}$ sunscreens of Sun Protection Factor (SPF)15,30, and 50 as UV ray blocking substances at the quantity of $2 \mathrm{ml}$ were tested. Testing the efficacy of the substances in this quantity would provide a perception of how the dandelion extract and sunscreens would perform at the level recommended for use. This is important because companies could be tremendously aided while creating their products by this information--especially if they are doing so with dandelion extract in the future as a result of such studies. This is due to the fact that the amount of extract needed for proper UV ray protection is unknown. In addition, a lack of data analysis was noted in the prior study, which is why the results were analyzed using statistical analyses such as Analysis of Variance (ANOVA) and Two Samples Assuming Equal Variance (t-test) in this experiment.

\section{- Methods}

The materials required for the experiment were dandelion extract with a concentration of $0.93 \mathrm{~g} / \mathrm{ml}$, SPF 15 Coppertone $\mathrm{T}^{\mathrm{TM}}$ sunscreen, SPF 30 Coppertone $^{\mathrm{TM}}$ sunscreen, SPF 50 Coppertone $^{\mathrm{TM}}$ sunscreen, ultraviolet (UV) sensitive paper, as well as common household Ziploc bags. Thirty individual sheets of UV sensitive paper were set aside per sunblocker, including the dandelion extract, amounting to 120 papers used and 30 trials. On each paper, $2 \mathrm{ml}$ of its respective sunblocker (either dandelion extract, Coppertone ${ }^{\mathrm{TM}}$ sunscreen Sun Protection Factor 15, Coppertone ${ }^{\mathrm{TM}}$ sunscreen Sun Protection Factor 30, or Coppertone ${ }^{\mathrm{TM}}$ sunscreen Sun Protection Factor 50) was spread evenly in an area with no direct sunlight. In addition, there were 30 other papers that were used as a control group upon which no sun blocking substance was smeared. Each paper was placed into its own individual plastic bag, taking care to not let the sun blocking substance smear onto the plastic of the bag's interior, as the trial wouldn't be fair if it occurred because the amount of the substance would no longer be equal. Once all 120 individual papers were smeared and put into their bags, they were left in the sunlight for fifteen minutes. All 30 trials were conducted at the same time, due to the fact that if they were not, there would be another factor to consider: the conditions outside, such as the temperature and humidity. Though the papers were left for approximately five minutes while the others were being smeared or being put into bags, the chemical reaction does not occur until placed directly in the sunlight. Care was taken to ensure that the papers were not overexposed, as this would disrupt the reaction taking place and nullify the trial.

The UV sensitive papers started out as a shade of blue, and once they were left in the sun with their sun blockers, a chemical reaction took place, causing the area around the smear (that was not smeared with the substance) to turn white. After 
15 minutes, the papers were withdrawn from their bags to an area with no direct sunlight, with caution being exercised so as not to touch the smeared portion of them, and were deposited into a bath of pure water in order to halt the chemical reaction taking place. For a full minute, each paper was kept in the bath. The papers were removed immediately once the minute was up to prevent discrepancies and overexposure to the water which could hinder the appearance of the actual reading of the UV light blockers. Each paper was then allowed to dry independently. Once they were dry, the un-smeared portions of the paper returned to the shade of blue they were prior to sun-exposure and the smeared portions showcased the UV protection each sun blocker had. A scale from 0-5 was used to grade each paper, with 0 being the best (lightest) and 5 being the worst (darkest), in terms of UV ray blocking (Table 1). Then, the average for each substance was calculated (Table 2). The papers were graded blindly in order to reduce bias. However, in order to identify which paper had been smeared with which sun blocking substance, a $\mathrm{D}, 15,30$, or 50 was written on the back of each paper according to the substance they'd been smeared with before they'd been smeared. The papers used in the control group had a $\mathrm{C}$ on the back.

Table 1 : Table containing the results of each individual paper as the grade given to them from $0-5$, with 0 being the best and 5 being the worst in terms of the ultraviolet protection they showcased.

\begin{tabular}{|c|c|c|c|c|}
\hline Sample no. & SPF 15 & SPF 30 & SPF 50 & Dandelion \\
\hline 1 & 4 & 3 & 3 & 1 \\
\hline 2 & 4 & 3 & 3 & 1 \\
\hline 3 & 4 & 3 & 3 & 1 \\
\hline 4 & 4 & 3 & 3 & 1 \\
\hline 5 & 5 & 3 & 3 & 1 \\
\hline 6 & 4 & 3 & 3 & 2 \\
\hline 7 & 5 & 3 & 3 & 1 \\
\hline 8 & 4 & 3 & 3 & 1 \\
\hline 9 & 4 & 4 & 3 & 1 \\
\hline 10 & 4 & 3 & 3 & 1 \\
\hline 11 & 4 & 3 & 2 & 2 \\
\hline 12 & 4 & 4 & 2 & 2 \\
\hline 13 & 4 & 3 & 3 & 1 \\
\hline 14 & 5 & 3 & 3 & 2 \\
\hline 15 & 4 & 3 & 3 & 1 \\
\hline 16 & 4 & 3 & 3 & 1 \\
\hline 17 & 4 & 3 & 3 & 1 \\
\hline 18 & 4 & 3 & 3 & 0 \\
\hline 19 & 4 & 3 & 3 & 2 \\
\hline 20 & 5 & 3 & 3 & 3 \\
\hline 21 & 4 & 3 & 2 & 1 \\
\hline 22 & 4 & 3 & 3 & 1 \\
\hline 23 & 4 & 3 & 3 & 1 \\
\hline 24 & 4 & 4 & 3 & 0 \\
\hline 25 & 4 & 3 & 3 & 1 \\
\hline 26 & 4 & 3 & 3 & 2 \\
\hline 27 & 4 & 3 & 3 & 1 \\
\hline 28 & 5 & 3 & 2 & 1 \\
\hline 29 & 4 & 3 & 3 & 1 \\
\hline 30 & 4 & 3 & 3 & 1 \\
\hline
\end{tabular}

Table 2 : Table containing the calculated averages of each of the sun blocking substances.

\begin{tabular}{|l|l|l|l|l|}
\hline Substance & SPF 15 & SPF 30 & SPF 50 & Dandelion \\
\hline Averages & 4.166 & 3.1 & 2.866 & 1.233 \\
\hline
\end{tabular}

\section{Results and Discussion}

The substance with the lowest amount of ultraviolet ray penetration turned out to be the dandelion extract, with an average of 1.233, and the substance with the highest level of UV ray penetration turned out to be the Sun Protection Factor (SPF) 15 Coppertone $^{\mathrm{TM}}$ sunscreen with 4.166 as an average (Figure 1). The SPF 30 Coppertone ${ }^{\mathrm{TM}}$ sunscreen had an average of 3.1 and the SPF 50 Coppertone ${ }^{\mathrm{TM}}$ sunscreen had an average of 2.866 (Figure 1). In essence, the bluer the smear was on the UV sensitive papers, the less efficient the sun-blocking substance was, meaning that more UV light was able to pass

Table 3 : Table showcasing all statistical analyses performed on the obtained data and their respective p-values, including their interpretation.

\begin{tabular}{|l|l|l|l|}
\hline $\begin{array}{l}\text { Type of Statistical } \\
\text { Analysis }\end{array}$ & Substances & P-value & Meaning \\
\hline ANOVA & All four tested blockers & $2.52 \mathrm{E}-56$ & $\begin{array}{l}\text { Significantly different } \\
(p<0.05)\end{array}$ \\
\hline t-test & SPF 15 and Dandelion & $1.04528 \mathrm{E}-35$ & $\begin{array}{l}\text { Significantly different } \\
(p<0.05)\end{array}$ \\
\hline t-test & SPF 30 and Dandelion & $2.543 \mathrm{E}-27$ & $\begin{array}{l}\text { Significantly different } \\
(p<0.05)\end{array}$ \\
\hline t-test & SPF 50 and Dandelion & $1.65803 \mathrm{E}-23$ & $\begin{array}{l}\text { Significantly different } \\
(p<0.05)\end{array}$ \\
\hline t-test & SPF 15 and SPF 30 & $1.15458 \mathrm{E}-17$ & $\begin{array}{l}\text { Significantly different } \\
(p<0.05)\end{array}$ \\
\hline t-test & SPF 15 and SPF 50 & $2.19028 \mathrm{E}-20$ & $\begin{array}{l}\text { Significantly different } \\
(p<0.05)\end{array}$ \\
\hline$t$-test & SPF 30 and SPF 50 & 0.160732192 & Similar ( $p>0.05)$ \\
\hline & & & \\
\hline
\end{tabular}

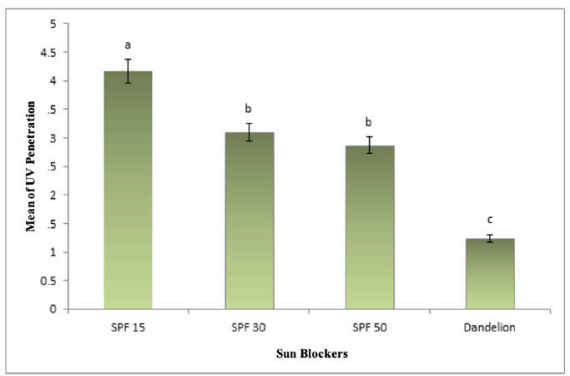

Figure 1 : Mean of UV penetration by different sun blockers tested. Bars represent mean $+\mathrm{SE}$ and the different letters indicate significant $(\mathrm{P}<0.05)$ differences among values obtained by performing statistical analyses.

through the substance. The results were analyzed by performing multiple statistical analyses, such as ANOVA and t-test.

The dandelion extract was found to have a significantly higher rate of UV protection $(\mathrm{p}<0.05)$ compared to all three other sunscreens tested (Table 3). The SPF 15 was concluded to possess significantly $(\mathrm{p}<0.05)$ lower rates of protection against UV rays. Even the SPF 50, the Coppertone ${ }^{\mathrm{TM}}$ sunscreen with the highest protection tested, had significantly lower UV ray protection compared to the dandelion extract. However, the SPF 30 and SPF 50 Coppertone ${ }^{\mathrm{TM}}$ sunscreens were very similar to each other in terms of UV protection. This is due to the fact that SPF 15 blocks $93 \%$ of ultraviolet irradiation, SPF 30 blocks 97\%, and SPF 50 blocks 98\%, leaving us with only a difference of one percent in SPF 30 and SPF 50's UV blocking. ${ }^{17}$ Thus, the results of this experiment suggest that dandelion extract exhibits UV ray protection of over $98 \%$. 
This study also suggests that depending on the amount of sunscreen used, UV ray protection increases or decreases, and that this principle applies to dandelion extract as well; the more substance applied to the UV ray sensitive papers, the more UV ray protection was granted. This conclusion was reached by comparing the results of a previous experiment on this topic in which 0.6 ounces of each substance was used instead of the $2 \mathrm{~mL}$ used in this experiment since the amount of sunblock recommended for use by experts is a mere nickel-sized amount and not 0.6 ounces. ${ }^{15,16}$ Companies and experts could keep this information in mind if they decide to develop a sunscreen utilizing dandelion extract to avoid the harmful effects that today's sunscreens have both on our health and that of our planet. They could use this information accordingly when recommending the quantity of their product that a consumer should use. It's worth noting that the papers were left in a backyard and though great care was taken to find an area that would receive the same amount of sunlight throughout the time period, a tree or animal may have cast a shadow over the papers unbeknownst to the researcher.

To expand on this topic, perhaps a different brand of sunscreen could be tested in comparison to the dandelion extract, and perhaps a study could be conducted on the effects of dandelion extract on coral and other forms of marine life; though it's natural, it may still have adverse effects on the undersea environment, and this is definitely something that could be further explored in the future. In addition, sunscreens with a Sun Protection Factor of over 50 could be tested, such as one with SPF 100 that blocks 99\% of UV rays, to get a more accurate perception of what percent of UV irradiation the dandelion extract blocks, and another study could be done in order to identify exactly what percentage of UV rays dandelion extract blocks. Through the findings obtained from this experiment that were further analyzed via ANOVA and t-test, it could be inferred that dandelion is a cleaner, healthier, and more efficient alternative to sunscreens, being that dandelion itself is natural, beneficial for human skin, and seems to possess superior UV ray protection compared to esteemed SPF 15, 30, and 50 Coppertone ${ }^{\mathrm{TM}}$ sunscreens, as seen in this study.

\section{Acknowledgements}

The author would like to acknowledge Maryam Bharucha from the Institute of Knowledge for her guidance.

\section{- References}

1. Statista Research Department. (2020, December 1). Sun Care Industry - Statistics \& Facts. Statista. https://www.statista.com/topics/1990/sun-care-industry/.

2. Ridder, M. (2020, November 23). Global market size of sunscreen cream 2019-2024. Statista. https://www.statista.com/statistics/866356/sunscreeningredients-global-market-size-forecast/.

3. Perry, A. (n.d.). Your Sunscreen Might Be Poisoning You. The Dr. Oz Show. Retrieved September 8, 2020, from https://www.doctoroz.com/article/yoursunscreen-might-be-poisoning-you.

4. What Is Oxybenzone and Why Is it in Sunscreen? (n.d.). Goddess Garden Organics. Retrieved November 7, 2019, from https://www.goddessgarden. com/what-is-oxybenzone-and-why-is-it-in-sunscreen/
$\% 2045 \% \mathrm{C} 2 \% \mathrm{~B} 0 \mathrm{C}$.

5. Yang, Y., \& Li, S. (2015, October 20). Dandelion Extracts Protect Human Skin Fibroblasts from UVB Damage and Cellular Senescence. Hindawi. https://www.hindawi.com/journals/ omc1/2015/619560/\#B15.

6. Treviño, J. (2018, July 16). Can We Create Sunscreen That Protects Both Humans and Coral Reefs? Smithsonian Magazine. https://www.smithsonianmag.com/ science/scientists-are-unraveling-new-dangerssunscreen-coral-reefs-180969627/.

7. Is Your Sunscreen Killing the Coral Reef? (2018, May 24). Ocean Conservancy. https://oceanconservancy.org/blog/2018/05/24/ sunscreen-killing-coral-reef/.

8. NOAA. (2021, February 26). Sunscreen Chemicals and Coral Reefs. National Oceanic and Atmospheric Association. https://oceanservice.noaa.gov/news/sunscreen-corals.html

9. Belluz, J. (2018, July 2). Hawaii is banning sunscreens that kill coral reefs. Vox. https://www.vox.com/2018/7/2/17525496/ hawaii-banning-sunscreen.

10. Calderone, J. (2019, February 7). The Truth About "Reef Safe" Sunscreen. Consumer Reports. https://www.consumerreports.org/sunscreens/thetruth-about-reef-safe-sunscreen/.

11. Schütz, K., Carle, R., \& Scheiber, A. (2006, October 11). Taraxacum-A review on its phytochemical and pharmacological profile. Science Direct. https://www.sciencedirect.com/science/article/ abs/pii/S0378874106003576.

12. Hill, A. (2018, July 18). 13 Potential Health Benefits of Dandelion. Healthline. https://www.healthline.com/ nutrition/dandelion-benefits.

13. Katja. (2019, March 21). Amazing Dandelion health and skin benefits. Wild for Nature. https://www.wildfornature.com/ dandelion-health-and-skin-benefits/.

14. Maria. (2017, January 2). Amazing Benefits of Dandelion for the Skin. TBOSC. https://thebestorganicskincare.com/amazingbenefits-of-dandelion-for-the-skin.

15. Soucar, J. (2015). It's More than a Weed, It's Sun Screen. CSEF. https://csef.usc.edu/History/2015/ Projects/J1923.pdf.

16. Hale, E. K. (2019, February 20). Ask the Expert: How Much Sunscreen Should I Be Using on My Face and Body? The Skin Cancer Foundation. https://www.skincancer.org/blog/ask-the-experthow-much-sunscreen-should-i-be-using-on-myface-and-body/.

17. Fitzpatrick, K. (2018, August 2). How Much SPF Do You Need In Your Sunscreen? UT News. https://news.utexas.edu/2018/06/06/how-much-spfdo-you-need-in-your-sunscreen/.

18. Bens, G. (2014). Sunscreens. SpringerLink. https://link.springer.com/chapter/10.1007/ 978-1-4939-0437-2_25? error=cookies_not_ supported \&code=e33f3c11-c732-4c5da4ec-6203b1858adf. 
19. Bhagat, J. (2019, November 19). 6 Side Effects Of Using Sunscreen You Should Be Aware Of. STYLECRAZE. https://www.stylecraze.com/ articles/side-effects-of-using-sunscreen/\#gref.

20. Chemical UVB+UVA Sunscreen/Sunblock: Octocrylene. (n.d.). SmartSkinCare.Com. https://www.smartskincare.com/skinprotection/ sunblocks/sunblock_octocylene.html.

21. Choi, U., Lee, O., Yim, J. H., Cho, C., Rhee, Y. K., Lim, S., \& Kim, Y. (2010, January 6). Hypolipidemic and Antioxidant Effects of Dandelion (Taraxacum officinale) Root and Leaf on Cholesterol-Fed Rabbits. MDPI. https:/www.mdpi.com/1422-0067/11/1/67

22. Czajka, K. (2019, April 18). Is Your Sunscreen Harming Coral Reefs? Pacific Standard. https://psmag.com/environment/is-your-sunscreenharming-coral-reefs.

23. Downs, C. A., Kramarsky-Winter, E., Segal, R., Fauth, J., Knutson, S., Bronstein, O., Ciner, F. R., Jeger, R., Lichtenfeld, Y., Woodley, C. M., Pennington, P., Caddenas, K., Kushmaro, A., \& Loya, Y. (2016, February). Toxicopathological Effects of the Sunscreen UV Filter, Oxybenzone (Benzophenone-3), on Coral Planulae and Cultured Primary Cells and Its Environmental Contamination in Hawaii and the U.S. Virgin Islands. PubMed.Gov. https://pubmed.ncbi.nlm.nih.gov/26487337/.

24. Environmental Working Group. (n.d.). EWG's 2020 Guide to Safer Sunscreens. EWG. https://www.ewg.org/sunscreen/report/thetrouble-with-sunscreen-chemicals/.

25. Finnerty, R. (2019, May 31). How Scientists Discovered the Link Between Sunscreen and Coral Reef Death. Hawaii Public Radio. https://www.hawaiipublicradio.org/post/howscientists-discovered-link-between-sunscreen-andcoral-reef-death\#stream $/ 0$.

26. Hfaiedh, M., Brahmi, D., \& Zourgui, L. (2014, October 1). Hepatoprotective effect of Taraxacum officinale leaf extract on sodium dichromateâinduced 1 iver injury in rats. Wiley Online Library. https://onlinelibrary.wiley.com/doi/abs/10.1002/tox.22048

27. Liebel, F., Kaur, S., Ruvulo, E., Kollias, N., \& Southall, M. D. (2012, July 1). Irradiation of Skin with Visible Light Induces Reactive Oxygen Species and MatrixDegrading Enzymes. ScienceDirect. https://www.sciencedirect.com/science/article/ pii/S0022202X15358292.

28. Mousa, M., Chatterjee, S. J., Ovadje, P., Hamm, C., \& Pandey, S. (2010, December 30). The Efficacy of Dandelion Root Extract in Inducing Apoptosis in Drug-Resistant Human Melanoma Cells. Hindawi. https://www.hindawi.com/journals/ecam/2011/129045/

29. National Center for Biotechnology Information. (n.d.). Octinoxate. PubChem. Retrieved October 17, 2020, from https://pubchem.ncbi.nlm.nih.gov/ compound/Octinoxate.

30. Pedroja, C. (2018, August 30). Octinoxate in Cosmetics: What You Should Know. Healthline. https://www.healthline.com/health/octinoxate.

31. Poljšak, B., \& Dahmane, R. (2012, February 29). Free Radicals and Extrinsic Skin Aging. Hindawi. https://www.hindawi.com/journals/drp/2012/135206/

32. Quan, T., Qin, Z., Xu, Y., He, T., Kang, S., Voorhees,

J. J., \& Fisher, G. J. (2010, June). Ultraviolet

Irradiation Induces CYR61/CCN1, a Mediator of

Collagen Homeostasis, through Activation of

Transcription Factor AP-1 in Human Skin Fibroblasts.

ScienceDirect.

https://www.sciencedirect.com/science/article/ pii/S0022202X15348508.

33. Sunscreen topical Side Effects. (n.d.). Drugs.Com. https://www.drugs.com/sfx/sunscreen-topical-sideeffects.html.

34. Tadimalla, R. D., \& Prabala, V., MD. (2019, December 19). Dandelions: Potential Benefits, Dosage, And Side Effects. STYLECRAZE. https://www.stylecraze.com/articles/amazing-benefitsof-dandelion-for-skin-hair-and-health/\#gref.

35. The Stream2Sea Marketing Team.

(2019, January 29). The Triple Threat: Oxybenzone, Octinoxate, and now Octocrylene. Stream2Sea. https://stream2sea.com/the-triple-threatoxybenzone-octinoxate-and-now-octocrylene/.

36. Thompson, D. (2019, May 6). Sunscreen Chemicals Enter Bloodstream at Potentially Unsafe Levels: Study. USNews. https://www.usnews.com/news/healthnews/articles/2019-05-06/sunscreen-chemicalsenter-bloodstream-at-potentially-unsafe-levels-study.

37. Transparency Market Research. (2018, December 17). Sun Care Market is Expected to Reach US\$24.9 Billion by 2024; Growing Awareness of Personal Care to Boost Global Market, Says TMR. Cision PR Newswire.

https://www.prnewswire.com/news-

releases/sun-care-market-is-expected-to-reach-us24-9-billion-by-2024-growing-awareness-ofpersonal-care-to-boost-global-marketsays-tmr-882935452.html.

38. U.S. Sun Care Market Size, Share | Industry Trends Report, 2018-2025. (2018, April). Grand View

Research.

https://www.grandviewresearch.com/industry-analysis/ us-sun-care-market.

39. Zachos, E., \& Rosen, E. (2019, May 21).

What sunscreens are best for you-and the planet? National Geographic.

https://www.nationalgeographic.com/travel/article/ sunscreen-destroying-coral-reefs-alternatives-travel-spd.

\section{Author}

Simra Mirza is currently a freshman at Institute of Knowledge. She enjoys working on science projects that can be applicable in our world and has won two first places in the Los Angeles County Science and Engineering Fair as well as an Honorable Mention in the national ExploraVision competition for her work. Besides her achievements in STEM, she has a love for public speaking and has been speaking in numerous gatherings of up to 400 attendees and has won a third place in the Global GiveLight Foundation Speech Competition. She is passionate about biological sciences and hopes to pursue a medical degree in the future 